\title{
An interplay between transcription, processing and degradation determines tRNA levels in yeast
}

\begin{tabular}{|r|l|}
\hline Journal: & WIREs RNA \\
\hline Manuscript ID: & RNA-571.R2 \\
\hline Wiley - Manuscript type: & Advanced Review \\
\hline Date Submitted by the Author: & $\mathrm{n} / \mathrm{a}$ \\
\hline Complete List of Authors: & $\begin{array}{l}\text { Wichtowska, Dominika; Institute of Biochemistry and Biophysics, Genetics } \\
\text { Burowski, Tomasz; Faculty of Chemistry, Warsaw University of Technology, } \\
\text { Boguta, Magdalena; Polish Academy of Sciences, Department of Genetics }\end{array}$ \\
\hline Keywords: & tRNA, yeast \\
\hline $\begin{array}{r}\text { Choose 1-3 topics to } \\
\text { categorize your article: }\end{array}$ & \begin{tabular}{l} 
tRNA Processing (RFAG) < RNA Processing (RFAA), Regulation of RNA \\
\hline
\end{tabular} \\
\hline
\end{tabular}

SCHOLARONE ${ }^{m}$

Manuscripts 


\begin{tabular}{|l|l|l|l|}
\hline$\square$ & Overview & $\begin{array}{l}\text { Overviews will provide a broad and relatively non-technical treatment of } \\
\text { important topics at a level suitable for advanced students and for researchers } \\
\text { without a strong background in the field. }\end{array}$ & $\begin{array}{l}5,000-8,000 \text { words } \\
\leq 16 \text { figures/tables } \\
50-100 \text { references }\end{array}$ \\
\hline$\square$ & $\begin{array}{l}\text { Advanced } \\
\text { Review }\end{array}$ & $\begin{array}{l}\text { Advanced Reviews, aimed at researchers and advanced students with a strong } \\
\text { background in the subject, will review key areas of research in a citation-rich } \\
\text { format similar to that of leading review journals. }\end{array}$ & $\begin{array}{l}4,000-6,000 \text { words } \\
\leq 10 \text { figures/tables } \\
50-75 \text { references }\end{array}$ \\
\hline$\square$ & $\begin{array}{l}\text { Focus } \\
\text { Article }\end{array}$ & $\begin{array}{l}\text { Focus articles are short articles, sometimes included within a larger article, } \\
\text { that describe specific real-world issues, examples, implementations, etc. } \\
\text { These articles will be technical in nature. }\end{array}$ & $\begin{array}{l}2,500-4,000 \text { words } \\
\leq 7 \text { figures/tables } \\
40-60 \text { references }\end{array}$ \\
\hline$\square$ & Opinion & $\begin{array}{l}\text { Opinions provide a forum for thought-leaders, hand-picked by the editors, to } \\
\text { provide a more individual perspective on the field in question. }\end{array}$ & $\begin{array}{l}2,000-4,000 \text { words } \\
\leq 5 \text { figures/tables } \\
30-60 \text { references }\end{array}$ \\
\hline
\end{tabular}

\title{
Article title: An interplay between transcription, processing and degradation determines tRNA levels in yeast
}

\author{
Dominika Wichtowska ${ }^{1}$, Tomasz W. Turowski ${ }^{1}$ and Magdalena Boguta ${ }^{*}$ \\ ${ }^{1}$ These authors contributed equally to this work \\ *Correspondence: magda@ibb.waw.pl \\ The authors declare no competing interests.
}

\begin{tabular}{|l|}
\hline First author: Dominika Wichtowska, Institute of Biochemistry and Biophysics, Polish \\
Academy of Sciences, Pawinskiego 5a, 02-106 Warsaw, Poland \\
\hline Second author: Tomasz W. Turowski, Institute of Biotechnology, Faculty of Chemistry, \\
Warsaw University of Technology, Noakowskiego 3, 00-664 Warsaw, Poland \\
\hline Third author: Magdalena Boguta, Institute of Biochemistry and Biophysics, Polish \\
Academy of Sciences, ul. Pawinskiego 5a, 02-106 Warsaw, Poland and Institute of \\
Biotechnology, Faculty of Chemistry, Warsaw University of Technology, Noakowskiego \\
3,00-664 Warsaw, Poland; email: magda@ibb.waw.pl \\
\hline
\end{tabular}

\section{Abstract}

tRNA biogenesis in yeast involves the synthesis of the initial transcript by RNA polymerase III followed by processing and controlled degradation in both the nucleus and the cytoplasm. A vast landscape of regulatory elements controlling tRNA stability in yeast has emerged from recent studies. Diverse pathways of tRNA maturation generate multiple stable and unstable intermediates. A significant impact on tRNA stability is exerted by a variety of nucleotide modifications. Pre-tRNAs are targets of exosome-dependent surveillance in the nucleus. Some tRNAs that are hypomodified or bear specific destabilising mutations are directed to the rapid tRNA decay (RTD) pathway leading to 5'-3' exonucleolytic degradation by Rat1 and Xrn1. Some tRNA molecules are marked for degradation by a double CCA at their $3^{\prime}$ ends. In addition, under different stress conditions, tRNA half-molecules 1 
can be generated by independent endonucleolytic cleavage events. Recent studies reveal unexpected relationships between the subsequent steps of tRNA biosynthesis and the mechanisms controlling its quality and turnover.

RNA levels are determined by two opposite processes: transcription and decay. In eukaryotic cells, the synthesis of mRNA, rRNA and tRNA are separately controlled in response to environmental conditions. Furthermore, each RNA category has unique processing, nucleus/cytoplasmic dynamics and decay pathways. However, the amount of final mature products must be matched to achieve the required translation efficiency. Transcription occurs in the nucleus, but RNA decay pathways operate in both the nucleus and the cytoplasm; these two processes are tightly coordinated by an unknown mechanism. A recent papers describe a mutual feedback between mRNA synthesis and degradation in Saccharomyces cerevisiae ${ }^{1-3}$, confirming earlier studies which reported that the processes are coupled $^{4,5}$. In general, impairing mRNA transcription increases mRNA stability. A similar relationship has been found for tRNA; repression of transcription by RNA polymerase III (Pol III) inhibits tRNA degradation in S. cerevisiae ${ }^{6}$.

A key player in RNA metabolism is the exosome, which participates in the $3^{\prime}$ end-maturation and/or quality control of almost every RNA molecule in the yeast cell. The remarkable impact of the exosome on tRNA turnover has recently been described. In a previous study, the degradation of hypomodified or misprocessed tRNAs was documented in yeast mutants defective in tRNA biosynthesis. Recent reports show that at least $50 \%$ of tRNA precursors are degraded in the absence of maturation defects ${ }^{7,8}$. These studies suggested that tRNA degradation by the exosome competes with normal processing. Selected data on the coupling between tRNA transcription, maturation and degradation in yeast are summarised in this review.

Box 1. tRNA transcription

tRNA precursors are transcribed by RNA polymerase III (Pol III). The Pol III apparatus consists of three complexes, the Pol III complex and the general factors TFIIIB and TFIIIC, which are required for transcription initiation and promoter recognition, respectively. The first step in transcription of a tRNA gene is the binding of TFIIIC to intragenic promoter elements. TFIIIC bound to the promoter recruits the TFIIIB complex upstream of the transcription initiation site. TFIIIB is composed of the TBP (TATA-binding protein) and the Brf1 and Bdp1 subunits ${ }^{9}$. The TFIIIB-DNA complex is sufficient to recruit the Pol III complex for multiple transcription cycles. The Pol III complex (0.7 MDa) comprises 17 subunits. The structural core of the complex is formed by nine subunits, and on the periphery of the core enzyme, Pol III contains eight additional subunits which form distinct subcomplexes involved in transcription initiation, elongation and termination (reviewed $\mathrm{in}^{10,11}$ ).

tRNA transcription is controlled in response to nutrient availability and other environmental circumstances by Maf1, a general and direct repressor that acts as an effector of several signalling pathways ${ }^{12}$. Pathways activated by growth-limiting conditions lead to dephosphorylation of Maf1 and importation into the nucleus. In the nucleus, hypophosphorylated Maf1 binds to the Pol III complex and prevents tRNA transcription. In favourable growth conditions, Maf1 is inactivated by phosphorylation, which operates at several levels to counteract Pol III repression ${ }^{13}$. The structure of the Maf1-Pol III co-complex has been determined by cryo-electron microscopy ${ }^{14}$. 
Box 2. tRNA maturation

tRNA processing involves multiple steps that occur in yeast in different subcellular locations (Fig. 1; for review see ${ }^{15,16}$ ). Initial transcripts are extended both at $5^{\prime}$ and $3^{\prime}$ termini and may contain introns. The $5^{\prime}$ leader is removed first from the initial transcripts by RNase $P$ endonuclease in the nucleolus. The processing of the $5^{\prime}$ leader generally precedes the trimming of the $3^{\prime}$ trailer, which is followed by CCA addition. End-processed tRNAs are transported to the cytoplasm by the exportin Los1. In yeast, ten tRNA families are encoded by intron-containing genes. The introns are removed from the end-processed transcripts by splicing machinery consisting of a heterotetrameric endonuclease bound to the outer surface of mitochondria, a cytoplasmic tRNA ligase and 2' phosphotransferase ${ }^{17,18}$. tRNAs are additionally modified throughout tRNA processing, both in the nucleus and in the cytoplasm. These modifications include alterations of ribose and the bases, e.g., methylation, isomerisation of uracil (U) to pseudouracil, conversion of uracil to dihydrouracil, conversion of adenosine to inosine, $\mathrm{N}$-acetylation of cytidine, conversion of guanosine to wybutosine or queuosine, isopentenylation of adenosine and methoxycarbonylmethylation and thiolylation of uracil $^{19,20}$. Numerous modifications of the anticodon loop have a major impact on the accuracy and efficacy of mRNA decoding ${ }^{21}$, while some tRNA body modifications contribute to its folding and stability ${ }^{22}$.

\section{STABILITY OF TRNA PRECURSORS}

\section{Diverse stability of intermediates generated by pre-tRNA processing}

Transcription by RNA polymerase III (Pol III; Box 1), followed by maturation of initial transcripts (Box 2 and Fig. 1), generates a variety of stable and unstable tRNA species. Some intermediates were only visible for particular tRNAs, while for others their abundance was below the detection level ${ }^{23}$. Deciphering whether the precursor level changes due to modulation of the processing rate or enhanced degradation can be a challenging task, especially due to the recently proposed competition between tRNA processing and degradation ${ }^{8}$. In this section, known mutations in the tRNA processing machinery that cause accumulation of pre-tRNA in yeast will be presented, emphasising those known to generate stable intermediates. The diverse intermediates of tRNA maturation accumulated in select yeast mutants are depicted in Figure 2 .

\section{5' leader cleavage}

The first step in tRNA maturation is $5^{\prime}$ leader cleavage by RNase $\mathrm{P}$, a multisubunit complex consisting in yeast of nine proteins and a single RNA encoded by the RPR1 gene ${ }^{24}$. In vivo studies of RNase $P$ functioning in yeast employ conditional mutants because all subunits are indispensable for cell viability. In a well-characterised thermo-sensitive mutant of the RPR1 gene, both immature unspliced and spliced pre-tRNA, containing extended $5^{\prime}$ and $3^{\prime}$ ends, accumulated at a restrictive temperature ${ }^{25}$. A lack of the expected precursor with only the $3^{\prime}$ end extended indicated that inhibition of $5^{\prime}$ end maturation also impaired the 3' processing (Fig. 2, lane 4). A similar accumulation of stable tRNA precursors was later detected in other mutants in $R P R 1^{26,27}$ and in genes encoding the 
protein subunits of RNase P: Pop $1^{28,29}$, Pop4 ${ }^{28,30}, \mathrm{Pop}^{31}{ }^{31}, \mathrm{Rpp} 1^{32}$, Pop5-Pop8 and Rpr2 ${ }^{32,33}$. Most of the RNase $P$ mutants, which accumulated tRNA transcripts with unprocessed ends, showed decreased levels of mature tRNAs and a lack of end-matured intron-containing forms after longer exposure to non-permissive conditions $s^{25,29,31,32}$. However, proper $5^{\prime}$ end processing of accumulated primary transcript was recovered when purified RNase $\mathrm{P}$ was added to the RNA extract from mutant cells, indicating that this form was stable ${ }^{25}$. These results indicate the importance of the first steps of tRNA processing for tRNA stability.

\section{3' trailer cleavage}

In the classical tRNA processing order, the $3^{\prime}$ trailer is removed following $5^{\prime}$ leader excision (for review see ${ }^{34}$ ); however, a reversed sequence of end maturation has been presented for pretRNA ${ }^{\operatorname{Tr} 35}$. The $3^{\prime}$ end maturation process requires more enzymes than does $5^{\prime}$ end trimming and the process is more complex.

Depending on the organism and the cellular compartment, $3^{\prime}$ processing of tRNA is achieved by exonucleolytic and/or endonucleolytic activities (as referred in ${ }^{36}$ ); in S. cerevisiae, two players have been identified: endonucelase Trz1 (an essential protein also known as RNase Z) and the 3'-5' exonuclease Rex1. The evidence for participation of Trz1 in 3' processing in vivo was recently confirmed (Skowronek and Kufel, personal communication).

Another protein required for $3^{\prime}$ maturation is Lhp1, a homologue of human La, which acts as a molecular chaperone for RNAs transcribed by Pol $\mathrm{III}^{37}$. Lhp1/La is the only protein involved in the early processing steps that is known to stabilise pre-tRNA by direct binding to the Pol III-termination track $\mathrm{UUU}_{\mathrm{OH}}{ }^{38}$. This direct binding likely helps maintain a conformation that facilitates endonucleolytic cleavage ${ }^{39}$.

Early studies of 3' maturation were performed in the sup61-10 mutant (G37 $\rightarrow A)$ in the gene encoding tRNA ${ }^{\text {Ser(GG) }}$. In this mutant, Lhp1 was required for endonucleolytic processing of the $3^{\prime}$ end and stabilisation of the altered structure of pre-tRNA ${ }^{\text {Ser(CGA) }}$. In the absence of Lhp1, the largest precursors of $\mathrm{TRNA} \mathrm{A}^{\mathrm{Ser}(\mathrm{CGA})}$ were shorter than in control cells and more abundant, and the same processing defect was observed for five out of nine other intron-containing tRNAs ${ }^{39}$. The viability of Ihp1 $\Delta$ cells suggested, however, that this mutant uses an alternative maturation pathway. Indeed, a pulse labelling experiment performed in the $/ h p 1 \Delta$ mutant indicated that the long $3^{\prime}$ trailer of pretRNA ${ }^{\text {Ser(CGA) }}$ was partially trimmed first; then, the $5^{\prime}$ leader was removed, followed by processing of the rest of the $3^{\prime}$ end. On the other hand, binding of Lhp1 to pre-tRNA $3^{\prime}$ trailers presumably protects them from exonuclease action ${ }^{39}$.

Lhp1 has also been implicated in the stabilisation of the fragile structure of tRNAs lacking particular modifications. First, overexpression of Lhp1 protein suppressed the temperature-sensitive (ts) phenotype of a trm 6 mutant lacking tRNA-1-methyladenosine 58 methyltransferase ${ }^{40}$. Second, negative genetic interactions of $I h p 1 \Delta$ and several mutations affecting tRNA modification were observed $^{41}$.

The 3'-5' exoribonuclease Rex1 was first shown to be required for maturation of a bicistrionic transcript of tRNA ${ }^{\mathrm{Arg} 3-\mathrm{Asp}} 42$, where the $\mathrm{UUU}_{\mathrm{OH}}$ tract is absent from tRNA ${ }^{\mathrm{Arg}}$ and Lhp1 cannot bind. The inability of Lhp1 to bind was assumed to be the reason for the accumulation of stable $3^{\prime}$-extended tRNA $^{\text {Arg }}$ in cells lacking Rex1. Similarly, initiator tRNA ${ }^{\text {Met }}$ with long $3^{\prime}$ trailers accumulated in rex1 $1 \Delta$ cells ${ }^{43}$ (Fig. 2, lane 6). Additionally, in the absence of Rex1, tRNA polyadenylation was increased, suggesting targeting of transcripts to the surveillance pathway (described in detail below). An 
analysis of pre-tRNA patterns in double and triple mutants lacking Rex1, Lhp1 and polyA polymerase Trf4 led Wolin and co-workers to conclude that Rex1 generates pre-tRNA processing intermediates, which can be subjected to nuclear surveillance ${ }^{44}$. Further, Lhp1 and Rex1 are known to compete with each other for interaction with some tRNA $3^{\prime}$ trailing sequences ${ }^{44}$. In a double deletion $\operatorname{lhp} 1 \Delta$ rex1 strain, the processing pattern of tRNA resembled the wild type arrangement; specifically, $5^{\prime}$ endmatured and $3^{\prime}$ end-extended forms with introns were visible. Additionally, the longer $3^{\prime}$ endunprocessed form of tRNA ${ }^{\text {Lys }}$ observed in rex1 $1 \Delta$ became slightly shorter when Lhp1 was absent (Fig. 2, lane 7). This indicated that Lhp1 binds nascent transcript to prevent 3' trimming by other exonucleases.

Finally, a complex of Lsm proteins is also involved in early processing, partially by interaction with Lhp $1^{45}$. Inactivation of a single Lsm protein from this complex resulted in the accumulation of stable $5^{\prime}$ and $3^{\prime}$ end-extended intron-containing precursors for all tRNAs tested and $5^{\prime}$ end-matured $3^{\prime}$ end-extended precursors for a subset (Fig. 2, lane 8). Following removal of the $3^{\prime}$ trailer from the original transcript, maturation of the $3^{\prime}$ end of tRNA requires the addition of a CCA sequence by nucleotidyl transferase ${ }^{16,46}$. CCA sequences in yeast are formed postranscriptionally in the nucleus and are required for tRNA aminoacylation.

\section{Coupling of Pol III transcription and pre-tRNA processing in the nucleus}

The coupling of Pol III transcription with early steps of pre-tRNA processing in yeast is still an open question. In contrast, human La protein, an ortholog of Lhp1, has been suggested by Maraia and co-workers to be involved in Pol III termination ${ }^{47}$. Additionally, a direct role for RNase P in Pol III transcription in mammalian cells has been shown ${ }^{48}$.

Earlier reports on the relationship between Pol III transcription and pre-tRNA processing in yeast focused on a dual role of Bdp1, an essential subunit of the TFIIIB factor ${ }^{49}$. Deletion of a small internal fragment of Bdp1 resulted in aberrant end-maturation of tRNA (Fig. 2, lane 3) and a thermosensitive (ts) growth phenotype. The RNA subunit of RNase P was clearly the limiting factor for $5^{\prime}$ end removal because RPR1 overexpression decreased the accumulation of the initial $5^{\prime}$ extended tRNA ${ }^{\text {Leu }}$ transcript and suppressed the growth defect. Moreover, Bdp1, directly or via other TFIIIB subunits, interacts physically with the RNase P complex, suggesting it plays a role in the coupling of Pol III transcription and pre-tRNA processing ${ }^{49}$.

tRNA processing in yeast is indirectly affected by the Pol III repressor Maf1. Both the initial transcripts and the end-processed, intron-containing tRNA precursors accumulated in the absence of Maf1 (Fig. 2, lane 2). Examination of the tRNA precursors in maf1 $\Delta$ cells by Northern hybridisation highlighted an imbalance between the rate of tRNA synthesis and the efficiency of tRNA maturation. Initial transcripts with immature ends accumulated particularly in maf1 $\Delta$ cells that experienced a shift to restrictive conditions ${ }^{50}$. Remarkably, this accumulation was not reversed by overexpression of the RPR1 gene, indicating that the RNA component of RNase $\mathrm{P}$ is unlikely to be limiting for the early steps of tRNA processing in the absence of Maf1. The interplay between Pol III activity and tRNA processing in the nucleus requires further study.

Pre-tRNA splicing 
According to the current model, the end maturation of intron-containing tRNAs in the nucleus precedes splicing, which, in yeast, occurs on the outer mitochondrial membrane ${ }^{17}$. Although there is evidence that tRNA precursors can undergo splicing before end maturation ${ }^{23,25,29,31,32}$, this pathway is presumably much slower and less efficient. All subunits of the splicing endonuclease complex are essential; this makes study difficult, and, consequently, only conditional mutants have been examined. One of these was the ts sen 2 mutant in the catalytic subunit of tRNA endonuclease. The sen 2 mutant accumulated a stable intermediate (2/3-exon with intron without other exon; designated on Fig. 2 as $\square$, lane 10 ), showing that splicing terminates at the $3^{\prime}$ splice site in vivo ${ }^{51,52}$ and in vitro ${ }^{17,53}$. Opposite accumulation of $2 / 3$ tRNA with impaired $3^{\prime}$ splice site cleavage was observed in vitro for mutations in another subunit, Sen34 $4^{53,54}$ (designated on Fig. 2 as $-\square$, lane 12). These data suggested that the Sen complex has two active sites, one responsible for $3^{\prime}$ and the other for $5^{\prime}$ cleavage $^{53}$ in the Sen complex.

The molecules accumulated in the sen2 mutant are thought to be stable, natural intermediates, which under normal conditions, would be further processed. This evidence comes from pulse-chase experiments where a shift to restrictive conditions and back to permissive conditions produced reversible accumulation of the splicing intermediate ${ }^{17}$.

Two other enzymes indispensable for splicing but not part of the Sen complex are the Trl1 ligase, which ligates the tRNA half-molecules, and tRNA 2'-phosphotransferase Tpt1, which catalyses transfer of the 2'-phosphate from the splice junction to NAD'. When the Trl1 ligase was missing, the endonucleolytically-cleaved but unligated tRNA half-molecules with mature ends accumulated as major pre-tRNA intermediates ${ }^{51,55}$ (Fig, 2, lane 9), and spliced-out introns of tRNA ${ }^{\text {Ile }}$ were more abundant ${ }^{55}$. Although a defect in Tpt1 did not cause obvious accumulation of unspliced pre-tRNA or half-molecules, spliced tRNAs with a $2^{\prime}$-phosphate in the splice junction were detected ${ }^{56}$. These data showed that Tpt1 is an essential component that is required for complete intron removal.

\section{Significance of nucleotide modifications for tRNA stability}

Apart from end-processing and splicing, tRNA maturation includes various modifications of nucleotides (box 2 ) which contribute to the relatively high stability of tRNA molecules. Approximately 100 different modifications of nucleotides occur in tRNA, with a range from 7 to 17 modifications per tRNA isotype in yeast ${ }^{15,57}$. Yeast cells lacking modifications in the anticodon loop often reveal severe growth phenotypes resulting from error-prone translation, while, usually, no obvious phenotypic effect is observed when a single modification in the tRNA body is missing ${ }^{15,21,58}$. Some tRNA body modifications contribute to its folding and stability ${ }^{59}$. Stabilising the tRNA structure by nucleotide modifications is a common strategy for all kingdoms of life; for instance, tRNAs of thermophilic organisms, which have a higher content of G.C base pairs, undergo modifications at elevated temperatures to increase their stability ${ }^{59}$. Several tRNA precursors and mature tRNAs, which are produced in yeast strains lacking particular tRNA modification enzymes, are known to be unstable due to a deficiency in specific modifications ${ }^{40,60,61}$. Studies on the turnover of improperly modified tRNAs have led to the identification of the tRNA decay pathways described below (Fig. 3).

\section{Pre-tRNA is a target of the TRAMP-mediated exosome-dependent surveillance pathway}

trm6 and trm61 mutants, unable to produce subunits of tRNA 1-methyladenosine 58 methyltransferase, are not viable at an elevated temperature due to the degradation of pre-tRNA ${ }_{i}^{\text {Met }}$ 
lacking $\mathrm{m}^{1} \mathrm{~A} 58^{40}$. The mechanism of $\mathrm{tRNA}_{\mathrm{i}}{ }^{\text {Met }}$ degradation was revealed by identification of spontaneous suppressors of the trm 6 phenotype as mutations in the RRP44 and TRF4 genes, which encode the core subunit of the exosome and a non-canonical poly(A) polymerase, respectively ${ }^{62}$. In addition, experimental evidence was obtained that the nascent pre-tRNA ${ }_{i}^{\text {Met }}$ transcript is targeted for polyadenylation by the TRAMP complex (파4/Air2/Mtr4 polyadenylation) and subsequent degradation by the exosome ${ }^{63}$. In the absence of Trf4 polyadenylation, pre-tRNA ${ }_{i}^{\text {Met }}$ can be restored by overexpression of the gene encoding $\operatorname{Trf5} 5^{63}$, likely because $\operatorname{Trf} 4$ and $\operatorname{Trf5}$ poly(A)-polymerases have partially overlapping substrate specificity ${ }^{64}$. Additionally, degradation of hypomodified $\operatorname{tRNA}_{i}{ }^{\text {Met }}$ is modulated by another TRAMP component, the DExH RNA helicase Mtr4 ${ }^{65,66}$. A recent model of TRAMP activity proposes that Trf4 extends the $3^{\prime}$ end, allowing the Mtr4 helicase to bind four or more $3^{\prime}$ adenylates ${ }^{67}$.

The exosome is a multiprotein complex that catalyses $3^{\prime} \rightarrow 5^{\prime}$ degradation of multiple RNA species $^{68}$. The main catalytic subunit of the exosome is Rrp44/Dis3, which has exo- and endonuclease activities. The exosome is present in both the nucleus and the cytoplasm. The nuclear exosome contains an additional catalytic subunit Rrp6 and associates with the TRAMP complex ${ }^{69,70}$. Degradation of pre-tRNA ${ }_{i}^{\text {Met }}$ lacking $\mathrm{m}^{1} \mathrm{~A} 58$ was assigned to the nuclear exosome because inactivation of Rrp6 was able to overcome the ts growth phenotype of the trm 6 mutation and partially stabilised tRNA $_{i}^{\text {Met } 62}$.

Importantly, the role of the nuclear exosome is not limited to degradation of hypomodified tRNAs, but it also includes the turnover of tRNA maturation byproducts in wild type cells. An indication that pre-tRNAs are normally degraded by the nuclear exosome came from the observation that their level is increased in mutants lacking the nuclear exosome subunit Rrp6 and cofactor Trf4. Although single rrp6 6 and $\operatorname{trf} 4 \Delta$ mutants did not clearly accumulate pre-tRNAs, loss of Rrp6 and Trf4 together strongly amplified this accumulation ${ }^{44}$.

A recent global analysis identified large amounts of pre-tRNAs associated with individual components of the exosome, despite the absence of processing defects ${ }^{7,8}$. In a mutants of Rrp44 or Rrp6 the relative numbers of various RNA products revealed designated Pol III transcripts as a major class of targets for the nuclear RNA surveillance machinery. The highly structured pre-tRNAs were enriched over pre-mRNAs among transcripts associated with Rrp6; these results were surprising, as pre-tRNAs are potentially less readily degradable than pre-mRNAs which usually lack strong secondary structure ${ }^{7}$.

While the mutation in Rrp44 exonuclease resulted in the accumulation of pre-tRNAs, the effect on the levels of mature tRNAs was controversial. Such a lack of effect seems consistent with reduced surveillance, rather than impaired processing of pre-tRNAs ${ }^{7,71}$. On the other hand, a relative increase in the level of mature tRNAs was detected in an independent study of mutants defective in Rrp44-exonuclease activity ${ }^{8}$, suggesting competition between processing and degradation of nascent pre-tRNAs. According to this study, the inefficient pre-tRNA degradation in the exosome mutant facilitated its maturation; conversely, inefficient maturation should direct pre-tRNAs to the exosome for degradation ${ }^{8}$.

tRNA precursors might be committed to degradation soon after transcription, possibly due to the interaction of the exosome with Pol III subunits and/or recognition of pre-tRNAs by the Nrd1Nab3 complex, which has been shown to favour their degradation ${ }^{71}$. Recent data suggest that wild type cells produce an excess of Pol III transcripts, which are normally degraded by the nuclear exosome ${ }^{8}$. As estimated by transcriptome-wide tiling microarrays and pulse-chase labelling of pre- 
tRNAs, more than $50 \%$ of nascent transcripts fail to generate functional tRNAs ${ }^{8}$. These results indicate that a major fraction of tRNA produced by Pol III in yeast cells is degraded.

\section{CONTROL OF MATURE tRNA STABILITY}

\section{Cleavage of mature tRNA generates half-molecules}

Another type of endonucleolytic cleavage of mature tRNAs, unrelated to splicing, has been observed in many organisms (for review see ${ }^{72}$ ). Endonucleolytic activity producing tRNA halfmolecules that were cleaved in the anticodon loop (Fig. 2, lane 13) was reported in yeast subjected to certain stress conditions such as oxidative stress, stationary phase, heat, and methionine or nitrogen starvation ${ }^{73}$. It seems that tRNA fragments are stable in unfavourable conditions, as they accumulate with prolonged exposure to stress, i.e., days in the stationary phase ${ }^{74}$. The endonuclease responsible for creating two halves of mature tRNAs is Rny $1^{72,75}$. The exosome appears not to be involved in the Rny1-dependent generation of half-tRNA molecules because these were also detected in cells deprived of exosome catalytic activity ${ }^{8}$.

Rny1-mediated cleavage was observed for a variety of tRNAs and is thus considered a general response. However, the biological significance of this response and the function of stable tRNA fragments in yeast cell are unclear. Endonucleolytic cleavage does not lead to a decrease in tRNA levels; although tRNA halves accumulate, the amount of mature tRNA does not change significantly ${ }^{73}$. The level of tRNA halves does not increase in mutants with tRNA processing defects, suggesting that cleavage is not a general tRNA quality control pathway. Intriguingly, studies in other organisms suggest the involvement of tRNA cleavage in the regulation of translation rate ${ }^{72}$. However, accumulation of cleaved tRNAs was not observed in yeast under conditions that lower the translation rate, arguing against this hypothesis ${ }^{73}$. Although numerous recent papers have reported a functional significance of tRNA-derived small RNAs in higher organisms ${ }^{76,77}$, it remains to be determined if and to what extent tRNA fragments regulate cellular functions in yeast.

\section{Mature tRNAs are degraded by the rapid tRNA decay (RTD) pathway}

Hypomodified and unstable tRNAs are rapidly degraded

Another pathway, the RTD pathway, is used by yeast to eliminate unstable mature tRNAs. Previous studies have indicated that hypomodified tRNAs are degraded at elevated temperatures in mutants lacking specific modification enzymes. Best described are tRNA ${ }^{\mathrm{Val}(\mathrm{AAC})}$ lacking $\mathrm{m}^{5} \mathrm{C}$ (5methylcytidine) and $\mathrm{m}^{7} \mathrm{G}$ (7-methylguanosine), synthesised in the trm4 $\operatorname{trm} 8 \Delta$ mutant $^{60}$, and

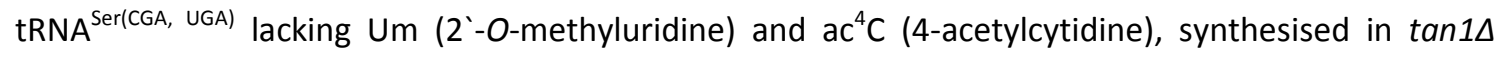
trm44 $\Delta$ cells $^{60,61}$. Degradation of these unstable, hypomodified tRNAs resulted in ts phenotypes,

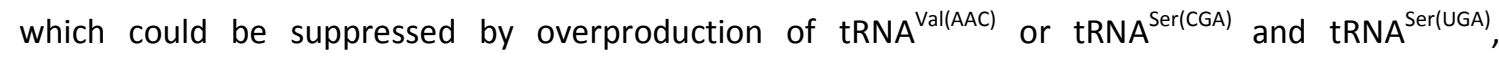
respectively. However, hypomodified tRNA is also degraded in trm8 8 , $\tan 1 \Delta$ and $\operatorname{trm} 1 \Delta$ mutants lacking thermo-sensitive growth phenotypes ${ }^{78}$. Calculation of the magnitude and rate of degradation $-25 \%$ of total cellular tRNA ${ }^{\text {Val(AAC) }}$ was degraded in 10 minutes following the temperature shift - led to the conclusion that the degraded hypomodified tRNA was mature ${ }^{60}$. Because the degradation was 
relatively fast, the new mechanism, discovered by E. Phizicky and co-workers, was called the RTD (rapid thNA decay) pathway.

To identify components of the RTD pathway, a genetic strategy was used that employed the ts growth phenotype of the trm4 4 trm $8 \Delta$ mutant. Both second-site suppressor and multicopy suppressor screens of the ts phenotype were performed ${ }^{6,78-80}$. By this approach, two $5^{\prime} \rightarrow 3^{\prime}$ exonucleases, Xrn1 and Rat1, were identified to be involved in the RTD-mediated degradation of hypomodified tRNA ${ }^{\text {Val(AAC) }}$, tRNA ${ }^{\text {Ser(CGA) }}$ and $\operatorname{tRNA}{ }^{\text {Ser(UGA) } 79,80}$. Additionally, purified Xrn1 selectively degraded the RTD substrate tRNAs in vitro ${ }^{22}$.

Data from the Phizicky lab suggested that RTD substrate recognition in vivo depends primarily on the instability of the acceptor stem and T stem, and not the anticodon stem ${ }^{22}$. A strong negative correlation was reported between the degradation by purified Xrn1 and the predicted stability of the acceptor and T-stems. Moreover, RTD-mediated degradation does not necessarily depend on a lack of modifications because fully modified tRNAs containing mutations that destabilise their structure are also degraded by RTD. These results suggest that tRNAs have evolved in eukaryotes to be structurally stable enough to resist degradation by $\mathrm{RTD}^{22}$.

Surprisingly, a hypomodified tRNA that is degraded by the RTD pathway is also targeted by TRAMP and degraded by the nuclear exosome ${ }^{60}$. In the trm $4 \Delta$ trm $8 \Delta$ strain, the level of tRNA ${ }^{\text {Vall(AAC) }}$ is partially restored by deletion of TRF4 or RRP6. In contrast, inactivation of Ski2, a cofactor of the cytoplasmic exosome, had no effect.

RTD-mediated tRNA degradation versus tRNA charging. CCACCA $3^{\prime}$ terminus marks tRNAs for RTD degradation

Observations of Phizicky's group argue that charged tRNA could also be a target of RTD as degradation of hypomodified tRNA is accompanied by tRNA deaminoacylation ${ }^{60,79}$. On the other hand, overproduction of valyl-tRNA synthetase suppresses the ts growth phenotype of trm4 $\operatorname{trm} 8 \Delta$, preventing RTD degradation of hypomodified tRNA ${ }^{\mathrm{Val}(\mathrm{AAC})}{ }^{6}$. Stabilisation of hypomodified tRNA is also provided by overexpression of eEF1A $A^{6,78}$, which is known to bind charged tRNAs ${ }^{81}$. Finally, uncharged hypomodified tRNA was a target of degradation by Xrn1 exonuclease in the in vitro system ${ }^{22}$.

tRNA charging requires CCA addition at the $3^{\prime}$ end. Remarkably, the tRNA ${ }^{\text {Ser }}$ species that are subjected to RTD also have an increased population of molecules with CCACCA or oligo(A) at the $3^{\prime}$ end instead of the usual CCA terminus ${ }^{82}$. CCACCA addition was more efficient for RTD-sensitive tRNAs, which have slightly destabilised acceptor stems. Surprisingly, the $x r n 1 \triangle$ strain with a defective RTD pathway showed significantly decreased levels of extended CCA and poly(A) tails ${ }^{82}$, provoking question about the relationship between the RTD pathway and the CCA-adding enzyme. Presumably, the CCA-adding enzyme can play a role in the quality control of tRNA by monitoring the acceptor stem.

Coupling of RTD, tRNA transcription and protein biosynthesis

Comprehensive screening for gene products that control the stability of hypomodified tRNA $^{\text {Val(AAC) }}$ has uncovered an indirect connection between RTD, tRNA transcription and protein biosynthesis. Two categories of gene products were identified as multicopy suppressors of the ts 
growth phenotype of the trm $4 \Delta$ trm $8 \Delta$ mutant: those affecting global tRNA transcription - Maf1 and truncated Pol III subunit Rpc160 - and those directly interacting with tRNA ${ }^{\text {Val(AAC) }}$ - the elongation factor eEF1A and valyl-tRNA synthetase ${ }^{6,50}$. All these suppressors stabilised hypomodified tRNA ${ }^{\text {Val(AAC) }}$.

The activity of Maf1 is known to be regulated by phosphorylation ${ }^{83-85}$. Significantly, the unphosphorylatable Maf1-7A variant, which exerts stronger Pol III repression and interacts with Pol III even under non-stress conditions, stabilised hypomodified tRNA ${ }^{\mathrm{Val}(\mathrm{AAC})}$ more efficiently than wild type Maf1 ${ }^{6}$. Based on prior data, similar suppressor effects on the trm $4 \Delta$ trm $8 \Delta$ growth defect were expected from Maf1 overexpression and effects from the $\mathrm{N}$-terminal part of Rpc160. Overexpression of a $5^{\prime}$-terminal fragment as well as point mutations in the RPC160 gene were identified previously as suppressors of the maf1 $\Delta$ growth phenotype, and they reduce tRNA levels in maf1 $1 \Delta$ cells ${ }^{86}$.

Along the same lines, inhibition of tRNA synthesis in a Maf1-independent manner, either by a point mutation in the Pol III subunit Rpc128 or decreased expression of the Rpc17 subunit, also suppressed the degradation of hypomodified tRNA ${ }^{\mathrm{Val}(\mathrm{AAC})}$. Regardless of the approach used, the reduced Pol III activity and consequent lower levels of total tRNA brought about a significant stabilisation of the hypomodified tRNA ${ }^{\text {Val(AAC). }}$

Suppression of the trm $4 \Delta$ trm $8 \Delta$ mutant growth phenotype was also achieved by overexpression of TEF1 or VAS1, encoding elongation factor eEF1A and valyl-tRNA synthetase, respectively. These proteins likely protect the hypomodified tRNA ${ }^{\mathrm{Val}(\mathrm{AAC})}$ by direct interactions ${ }^{6}$. Evidence that elongation factor eEF1A competes with the RTD pathway for substrate tRNAs was also presented in an independent study by the Phizicky group ${ }^{78}$. Moreover overexpression of seryl-tRNA synthetase partially rescues ts phenotype of $\tan 1 \Delta \operatorname{trm} 44 \Delta$ expressing hypomodified $\operatorname{tRNA}{ }^{\text {Ser(CGA) }}$ and $\operatorname{tRNA} A^{\text {Ser(UGA) }}$ 87 .

In conclusion, two alternative mechanisms have been proposed by which RTD may be limited: a decrease in tRNA synthesis or an increase in the levels of specific tRNA-interacting proteins that protect unstable tRNA against degradation. According to the proposed model, mature tRNAs compete with each other for interacting proteins and thus the availability of these proteins becomes limiting. Due to the missing $\mathrm{m}^{7} \mathrm{G}_{46}$ and $\mathrm{m}^{5} \mathrm{C}_{49}$ modifications, the tertiary structure of the acceptor and T stems in tRNA ${ }^{\text {Val(AAC) }}$ is imperfect or less stable (references in ${ }^{22}$ ), and therefore the interaction of this tRNA with proteins is compromised. In other words, it would be a poorer competitor for valyltRNA synthetase or eEF1A than other tRNAs. Globally decreased tRNA synthesis would relieve this competition by decreasing the overall tRNA:protein ratio, thereby allowing even the imperfect, hypomodified tRNA ${ }^{\mathrm{Val}(\mathrm{AAC})}$ to find interaction protein(s). Overexpression of limiting protein partner(s) would reduce the tRNA:protein ratio as well and, consequently, produce the same effect. We suggest that this explains the suppression of tRNA ${ }^{\text {Val(AAC) }}$ degradation by valyl-tRNA synthetase and eEF1A.

\section{Conclusion}

Substantial progress has been made in the past decade to delineate the mechanisms controlling tRNA levels and quality. Coordination of tRNA and ribosome functions requires regulation of tRNA levels in response to environmental information. On the other hand, the structural and functional integrity of tRNA is obligatory for translation fidelity. Recent studies indicate that optimisation of tRNA control is achieved by a network of processing steps that follow tRNA biosynthesis (Fig. 4). 
The rate of tRNA transcription by Pol III must be adjusted to the efficiency of the processing machinery ${ }^{87}$. An imbalance between the rates of tRNA synthesis and the efficiency of its maturation, observed in cells lacking the Pol III repressor Maf1, resulted in the accumulation of variable tRNA maturation intermediates ${ }^{50}$. It remains to be seen whether any step of early tRNA processing in yeast is controlled in a co-transcriptional manner.

Additional fascinating aspects of extended Pol III control involve tRNA decay. In wild type cells, a large portion of newly transcribed pre-tRNA is eliminated by the nuclear exosome, possibly due to its interaction with Pol III subunits ${ }^{8}$. According to the proposed model, the processing of newly transcribed tRNA competes with its degradation by the nuclear exosome ${ }^{7,8}$. Further studies are required to determine exactly how the exosome is associated with the Pol III complex. An indirect effect of Pol III transcription on the turnover of mature tRNAs, which are subject to rapid tRNA decay (RTD), was also detected. Some hypomodified tRNAs, or those bearing specific destabilising mutations, are directed to the RTD pathway, leading to their 5'-3' exonucleolytic degradation by Rat1 and Xrn1. RTD is prevented by Pol III inhibition, as hypomodified tRNA is stabilised by mutations which decrease the global tRNA level ${ }^{6}$.

tRNA maturation occurs in various cellular compartments. Moreover, tRNA can travel in a retrograde direction from the cytoplasm to the nucleus. The subcellular distribution of tRNA seems to serve unanticipated functions in diverse processes, including responses to nutrient availability, DNA repair and HIV replication ${ }^{15}$. Both export and retrograde import of tRNA to the nucleus are controlled by signalling pathways ${ }^{88,89}$. Maf1-mediated Pol III transcription control and tRNA nuclear export are regulated by environmental conditions in a coordinated manner ${ }^{50}$. Although dynamic control of tRNA modifications during cellular stress has been studied by a quantitative systems approach $^{90}$, the regulation of tRNA processing by cell physiology has not been addressed experimentally so far. However, such control has been shown for mRNA maturation ${ }^{91}$.

Several links exist between tRNA metabolism and translation. One interesting aspect is the inhibition of translation mediated by the Gcn4 factor in response to accumulation of immature tRNAs in the nucleus $5^{50,92,93}$. This response is independent of the phosphorylation of elF2 $\alpha$ by Gcn2 kinase, suggesting an unknown molecular mechanism. Another newly reported connection with translation is the decay of mature tRNAs. Experiments have shown that eukaryotic elongation factor $1 \mathrm{~A}$ (eEF1A) competes with the RTD pathway for tRNA substrates ${ }^{6,78}$. Further experiments will be required to clarify how the RTD pathway competes with eEF1A for specific tRNA substrates and how RTD interacts with other components of the translation machinery and other cellular processes.

Most of the recent progress in deciphering the cell biology of eukaryotic tRNA biogenesis and its response to physiological events has been achieved by studies in yeast. Future studies are required to learn whether the networking of tRNA synthesis, maturation and turnover operates in higher eukaryotes as well.

\section{Perspectives}

Within the past decade, there has been much progress to delineate the mechanisms by which tRNA levels are controlled. These studies primarily concentrated on the regulation of Pol III-mediated tRNA transcription and cellular dynamics in response to environmental signals. It should be noted that in the past decade, the dogma of tRNA stability has been overturned, and multiple pathways for tRNA 
degradation have been discovered. The interplay between transcription and degradation has raised many questions, some of which have been outlined in this review. However, new questions have been raised concerning a direct interaction of the Pol III complex (or Pol III functional module) with the nuclear exosome and Pol III assistance in tRNA degradation. The newly discovered bidirectional trafficking of tRNA likely serves as a tRNA quality control mechanism. It would be interesting to determine if there is an interplay between tRNA dynamics and decay. Powerful yeast genetics and molecular biology will contribute towards an understanding of the networks involved in tRNA regulation pathways.

\section{Figure captions}

Figure 1. Maturation of intron containing tRNA. After transcription by Pol III subsequent steps of end maturation take place in the nucleus, next precursor is exported to the cytoplasm where intron is spliced, tRNA can be charged and directed to further processes. Enzymes responsible for each processing step are listed above corresponding arrows. Modifications which can be added in each step of tRNA biosynthesis are not presented on the scheme.

Figure 2. Representation of forms and fragments of precursors and mature tRNAs that accumulated or appeared in yeast mutants or under specific conditions. An example of Northern analysis of

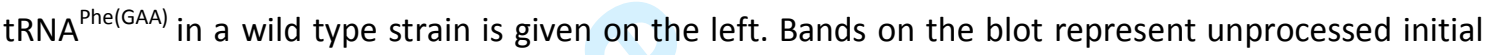
transcripts (designated $\square-\square$ ), 5' end matured intron-containing pre-tRNAs (designated $\square--$ ), endmatured intron-containing pre-tRNAs (designated $\square$ ) and mature tRNAs (designated $\square$ ). A graphical version of Northern blots reported previously for mutants defective in tRNA biosynthesis displays various intermediate forms designated by the respective symbols. Statement "not shown" is used to underline the forms that are not visualised by the given probes on Northern blots from cited articles. The band size is proportional to the wild type, in exception of those that are from in vitro studies. Each lane represents pre-tRNA and tRNA forms and the amount of each found in defined mutants as following: Maf1 -tRNA ${ }^{\text {Phe(GAA) }}$ in ${\text { maf1 } 1 \Delta^{50} \text {; Bdp1- tRNA }}^{\text {Ile(UAU) }}$ in bdp1 $\Delta 253-269^{49}$; RNase P tRNA ${ }^{\text {Leu(CAA) }}$ at $38^{\circ} \mathrm{C}$ in RPR1 cDNA dimer ${ }^{25}$; Lhp1- tRNA ${ }^{\text {Tyr (GUA) }}$ in Ihp1 $\Delta^{44}$; Rex1- tRNA ${ }^{\text {Lys(UUU) }}$ in rex1 $1 \Delta^{44}$; Lhp1 and Rex1- tRNA ${ }^{\text {Lys(UUU) }}$ in Ihp1 $\operatorname{rex} 1 \Delta^{44}$; Lsm- tRNA ${ }^{\text {Leu(CAA) }}$ after $6 \mathrm{~h}$ in glucose in GAL::Ism $3^{35}$; Sen2- tRNA ${ }^{\text {Leul(CAA) }}$ in sen2-3 $3^{52}$; Sen2- tRNA ${ }^{\text {Tyr }}$ in Sen2 (His297Ala), in vitro study ${ }^{53}$; Sen34- tRNA ${ }^{\text {Tyr }}$ in Sen34 (His217Ala) in vitro study ${ }^{53}$, Trl1- tRNA ${ }^{\text {Leu(CAA) }}$ in trl1-4 with empty vector at $37^{\circ} \mathrm{C}^{51}$, stress induced cleavage- tRNA ${ }^{\text {His(GTG) }}$ in $h$ ts1.1 after 15 min at $39^{\circ} \mathrm{C}^{73}$.

Figure 3. Current model of degradation during tRNA lifecycle. Newly transcribed tRNA precursor (pre-tRNA) can be polyadenylated on the $3^{\prime}$ end by TRAMP ( $\underline{\operatorname{Tr}} 4$ /Air2/느tr4 polyadenylation) complex and targeted to degradation by exosome. During early steps of processing defective intermediate can undergo exosomal degradation after marking with poly A tail. If the particle is unstable it can be marked with additional CCA sequence and directed to the Rapid tRNA Decay (RTD) pathway and/or polyadenylated and degraded by the exosome. As relationships of additional CCA marking of tRNA with RTD or exosome and correlation between exosome and RTD are unclear, they are marked on the scheme with dashed green lines. Lack of some modifications or mutations causing structural defects of mature tRNA results in instability of tRNA particle which is directed to RTD pathway. How mature stable tRNA is directed to degradation is still unknown. 
1. Sun, M. et al. Comparative dynamic transcriptome analysis (CDTA) reveals mutual feedback between mRNA synthesis and degradation. Genome Res. 22, 1350-1359 (2012).

2. Haimovich, G. et al. Gene expression is circular: factors for mRNA degradation also foster mRNA synthesis. Cell 153, 1000-1011 (2013).

3. Harel-Sharvit, L. et al. RNA polymerase II subunits link transcription and mRNA decay to translation. Cell 143, 552-563 (2010).

4. Bregman, A. et al. Promoter elements regulate cytoplasmic mRNA decay. Cell 147, 1473-1483 (2011).

5. Shalem, O., Groisman, B., Choder, M., Dahan, O. \& Pilpel, Y. Transcriptome kinetics is governed by a genome-wide coupling of mRNA production and degradation: a role for RNA Pol II. PLOS Genet. 7, e1002273 (2011).

6. Turowski, T. W., Karkusiewicz, I., Kowal, J. \& Boguta, M. Maf1-mediated repression of RNA polymerase III transcription inhibits tRNA degradation via RTD pathway. RNA 18, 1823-1832 (2012).

7. Schneider, C., Kudla, G., Wlotzka, W., Tuck, A. \& Tollervey, D. Transcriptome-wide Analysis of Exosome Targets. Mol. Cell (2012). doi:10.1016/j.molcel.2012.08.013

8. Gudipati, R. K. et al. Extensive Degradation of RNA Precursors by the Exosome in Wild-Type Cells. Mol. Cell (2012). doi:10.1016/j.molcel.2012.08.018

9. Acker, J., Conesa, C. \& Lefebvre, O. Yeast RNA polymerase III transcription factors and effectors. Biochim. Biophys. Acta 1829, 283-295 (2013).

10. Vannini, A. A structural perspective on RNA polymerase I and RNA polymerase III transcription machineries. Biochim. Biophys. Acta 1829, 258-264 (2013).

11. Upadhya, R., Lee, J. \& Willis, I. M. Maf1 is an essential mediator of diverse signals that repress RNA polymerase III transcription. Mol. Cell 10, 1489-1494 (2002).

12. Boguta, M. Maf1, a general negative regulator of RNA polymerase III in yeast. Biochim. Biophys. Acta 1829, 376-384 (2013).

13. Towpik, J., Graczyk, D., Gajda, A., Lefebvre, O. \& Boguta, M. Derepression of RNA polymerase III transcription by phosphorylation and nuclear export of its negative regulator, Maf1. J. Biol. Chem. 283, 17168-17174 (2008).

14. Vannini, A. et al. Molecular basis of RNA polymerase III transcription repression by Maf1. Cell 143, 59-70 (2010).

15. Phizicky, E. M. \& Hopper, A. K. tRNA biology charges to the front. Genes Dev. 24, 1832-1860 (2010). 
16. Hopper, A. K. Transfer RNA Post-Transcriptional Processing, Turnover, and Subcellular Dynamics in the Yeast Saccharomyces cerevisiae. Genetics 194, 43-67 (2013).

17. Yoshihisa, T., Ohshima, C., Yunoki-Esaki, K. \& Endo, T. Cytoplasmic splicing of tRNA in Saccharomyces cerevisiae. Genes Cells 12, 285-297 (2007).

18. Shaheen, H. H. \& Hopper, A. K. Retrograde movement of tRNAs from the cytoplasm to the nucleus in Saccharomyces cerevisiae. Proc. Natl. Acad. Sci. U.S.A. 102, 11290-11295 (2005).

19. Hopper, A. K. \& Phizicky, E. M. tRNA transfers to the limelight. Genes Dev. 17, 162-180 (2003).

20. Hopper, A. K., Pai, D. A. \& Engelke, D. R. Cellular dynamics of tRNAs and their genes. FEBS Lett. 584, 310-317 (2010).

21. Grosjean, H., de Crécy-Lagard, V. \& Marck, C. Deciphering synonymous codons in the three domains of life: co-evolution with specific tRNA modification enzymes. FEBS Lett. 584, 252-264 (2010).

22. Whipple, J. M., Lane, E. A., Chernyakov, I., D'Silva, S. \& Phizicky, E. M. The yeast rapid tRNA decay pathway primarily monitors the structural integrity of the acceptor and T-stems of mature tRNA. Genes Dev. 25, 1173-1184 (2011).

23. O'Connor, J. P. \& Peebles, C. L. In vivo pre-tRNA processing in Saccharomyces cerevisiae. Mol. Cell. Biol. 11, 425-439 (1991).

24. Xiao, S., Houser-Scott, F. \& Engelke, D. R. Eukaryotic ribonuclease P: increased complexity to cope with the nuclear pre-tRNA pathway. J. Cell. Physiol. 187, 11-20 (2001).

25. Lee, J. Y., Rohlman, C. E., Molony, L. A. \& Engelke, D. R. Characterization of RPR1, an essential gene encoding the RNA component of Saccharomyces cerevisiae nuclear RNase P. Mol. Cell. Biol. 11, 721-730 (1991).

26. Ziehler, W. A., Morris, J., Scott, F. H., Millikin, C. \& Engelke, D. R. An essential protein-binding domain of nuclear RNase P RNA. RNA 7, 565-575 (2001).

27. Marvin, M. C. et al. Accumulation of noncoding RNA due to an RNase P defect in Saccharomyces cerevisiae. RNA 17, 1441-1450 (2011).

28. Hiley, S. L., Babak, T. \& Hughes, T. R. Global analysis of yeast RNA processing identifies new targets of RNase III and uncovers a link between tRNA $5^{\prime}$ end processing and tRNA splicing. Nucleic Acids Res. 33, 3048-3056 (2005).

29. Lygerou, Z., Mitchell, P., Petfalski, E., Séraphin, B. \& Tollervey, D. The POP1 gene encodes a protein component common to the RNase MRP and RNase P ribonucleoproteins. Genes Dev. 8, 1423-1433 (1994).

30. Chu, S., Zengel, J. M. \& Lindahl, L. A novel protein shared by RNase MRP and RNase P. RNA 3, 382-391 (1997).

31. Dichtl, B. \& Tollervey, D. Pop3p is essential for the activity of the RNase MRP and RNase $P$ ribonucleoproteins in vivo. EMBO J. 16, 417-429 (1997).

32. Stolc, V. \& Altman, S. Rpp1, an essential protein subunit of nuclear RNase P required for processing of precursor tRNA and 35S precursor rRNA in Saccharomyces cerevisiae. Genes Dev. 11, 2926-2937 (1997).

33. Chamberlain, J. R., Lee, Y., Lane, W. S. \& Engelke, D. R. Purification and characterization of the nuclear RNase P holoenzyme complex reveals extensive subunit overlap with RNase MRP. Genes Dev. 12, 1678-1690 (1998).

34. Maraia, R. J. \& Lamichhane, T. N. 3' processing of eukaryotic precursor tRNAs. Wiley Interdiscip Rev RNA 2, 362-375 (2011).

35. Kufel, J. \& Tollervey, D. 3'-processing of yeast tRNATrp precedes 5'-processing. RNA 9, 202-208 (2003).

36. Daoud, R., Forget, L. \& Lang, B. F. Yeast mitochondrial RNase P, RNase $Z$ and the RNA degradosome are part of a stable supercomplex. Nucleic Acids Res. 40, 1728-1736 (2012).

37. Chakshusmathi, G., Kim, S. D., Rubinson, D. A. \& Wolin, S. L. A La protein requirement for efficient pre-tRNA folding. EMBO J. 22, 6562-6572 (2003). 
38. Lin-Marq, N. \& Clarkson, S. G. A yeast RNA binding protein that resembles the human autoantigen La. J. Mol. Biol. 245, 81-85 (1995).

39. Yoo, C. J. \& Wolin, S. L. The yeast La protein is required for the $3^{\prime}$ endonucleolytic cleavage that matures tRNA precursors. Cell 89, 393-402 (1997).

40. Anderson, J. et al. The essential Gcd10p-Gcd14p nuclear complex is required for 1methyladenosine modification and maturation of initiator methionyl-tRNA. Genes Dev. 12, 36503662 (1998).

41. Copela, L. A., Chakshusmathi, G., Sherrer, R. L. \& Wolin, S. L. The La protein functions redundantly with tRNA modification enzymes to ensure tRNA structural stability. RNA 12, 644654 (2006).

42. Van Hoof, A., Lennertz, P. \& Parker, R. Three conserved members of the RNase D family have unique and overlapping functions in the processing of $5 \mathrm{~S}, 5.8 \mathrm{~S}, \mathrm{U} 4, \mathrm{U} 5, \mathrm{RN}$ ase MRP and RNase $\mathrm{P}$ RNAs in yeast. EMBO J. 19, 1357-1365 (2000).

43. Ozanick, S. G. et al. Rex1p deficiency leads to accumulation of precursor initiator tRNAMet and polyadenylation of substrate RNAs in Saccharomyces cerevisiae. Nucleic Acids Res. 37, 298-308 (2009).

44. Copela, L. A., Fernandez, C. F., Sherrer, R. L. \& Wolin, S. L. Competition between the Rex1 exonuclease and the La protein affects both Trf4p-mediated RNA quality control and pre-tRNA maturation. RNA 14, 1214-1227 (2008).

45. Kufel, J., Allmang, C., Verdone, L., Beggs, J. D. \& Tollervey, D. Lsm proteins are required for normal processing of pre-tRNAs and their efficient association with La-homologous protein Lhp1p. Mol. Cell. Biol. 22, 5248-5256 (2002).

46. Aebi, M. et al. Isolation of a temperature-sensitive mutant with an altered tRNA nucleotidyltransferase and cloning of the gene encoding tRNA nucleotidyltransferase in the yeast Saccharomyces cerevisiae. J. Biol. Chem. 265, 16216-16220 (1990).

47. Fan, H. et al. Phosphorylation of the human La antigen on serine 366 can regulate recycling of RNA polymerase III transcription complexes. Cell 88, 707-715 (1997).

48. Reiner, R., Ben-Asouli, Y., Krilovetzky, I. \& Jarrous, N. A role for the catalytic ribonucleoprotein RNase P in RNA polymerase III transcription. Genes Dev. 20, 1621-1635 (2006).

49. Ishiguro, A., Kassavetis, G. A. \& Geiduschek, E. P. Essential roles of Bdp1, a subunit of RNA polymerase III initiation factor TFIIIB, in transcription and tRNA processing. Mol. Cell. Biol. 22, 3264-3275 (2002).

50. Karkusiewicz, I. et al. Maf1 protein, repressor of RNA polymerase III, indirectly affects tRNA processing. J. Biol. Chem. 286, 39478-39488 (2011).

51. Dhungel, N. \& Hopper, A. K. Beyond tRNA cleavage: novel essential function for yeast tRNA splicing endonuclease unrelated to tRNA processing. Genes Dev. 26, 503-514 (2012).

52. Ho, C. K., Rauhut, R., Vijayraghavan, U. \& Abelson, J. Accumulation of pre-tRNA splicing '2/3' intermediates in a Saccharomyces cerevisiae mutant. EMBO J. 9, 1245-1252 (1990).

53. Trotta, C. R., Paushkin, S. V., Patel, M., Li, H. \& Peltz, S. W. Cleavage of pre-tRNAs by the splicing endonuclease requires a composite active site. Nature 441, 375-377 (2006).

54. Trotta, C. R. et al. The yeast tRNA splicing endonuclease: a tetrameric enzyme with two active site subunits homologous to the archaeal tRNA endonucleases. Cell 89, 849-858 (1997).

55. Phizicky, E. M., Consaul, S. A., Nehrke, K. W. \& Abelson, J. Yeast tRNA ligase mutants are nonviable and accumulate tRNA splicing intermediates. J. Biol. Chem. 267, 4577-4582 (1992).

56. Spinelli, S. L., Malik, H. S., Consaul, S. A. \& Phizicky, E. M. A functional homolog of a yeast tRNA splicing enzyme is conserved in higher eukaryotes and in Escherichia coli. Proc. Natl. Acad. Sci. U.S.A. 95, 14136-14141 (1998).

57. Phizicky, E. M. \& Alfonzo, J. D. Do all modifications benefit all tRNAs? FEBS Lett. 584, 265-271 (2010). 
58. El Yacoubi, B., Bailly, M. \& de Crécy-Lagard, V. Biosynthesis and function of posttranscriptional modifications of transfer RNAs. Annu. Rev. Genet. 46, 69-95 (2012).

59. Motorin, Y. \& Helm, M. tRNA stabilization by modified nucleotides. Biochemistry 49, 4934-4944 (2010).

60. Alexandrov, A. et al. Rapid tRNA decay can result from lack of nonessential modifications. Mol. Cell 21, 87-96 (2006).

61. Kotelawala, L., Grayhack, E. J. \& Phizicky, E. M. Identification of yeast tRNA Um(44) 2'-Omethyltransferase (Trm44) and demonstration of a Trm44 role in sustaining levels of specific tRNA(Ser) species. RNA 14, 158-169 (2008).

62. Kadaba, S. et al. Nuclear surveillance and degradation of hypomodified initiator tRNAMet in S. cerevisiae. Genes Dev. 18, 1227-1240 (2004).

63. Kadaba, S., Wang, X. \& Anderson, J. T. Nuclear RNA surveillance in Saccharomyces cerevisiae: Trf4p-dependent polyadenylation of nascent hypomethylated tRNA and an aberrant form of $5 \mathrm{~S}$ rRNA. RNA 12, 508-521 (2006).

64. San Paolo, S. et al. Distinct roles of non-canonical poly(A) polymerases in RNA metabolism. PLoS Genet. 5, e1000555 (2009).

65. Jia, H. et al. The RNA helicase Mtr4p modulates polyadenylation in the TRAMP complex. Cell 145, 890-901 (2011).

66. Wang, X., Jia, H., Jankowsky, E. \& Anderson, J. T. Degradation of hypomodified tRNA(iMet) in vivo involves RNA-dependent ATPase activity of the DExH helicase Mtr4p. RNA 14, 107-116 (2008).

67. Jia, H., Wang, X., Anderson, J. T. \& Jankowsky, E. RNA unwinding by the Trf4/Air2/Mtr4 polyadenylation (TRAMP) complex. Proc. Natl. Acad. Sci. U.S.A. 109, 7292-7297 (2012).

68. Chlebowski, A., Tomecki, R., López, M. E. G., Séraphin, B. \& Dziembowski, A. Catalytic properties of the eukaryotic exosome. Adv. Exp. Med. Biol. 702, 63-78 (2011).

69. Schaeffer, D., Clark, A., Klauer, A. A., Tsanova, B. \& van Hoof, A. Functions of the cytoplasmic exosome. Adv. Exp. Med. Biol. 702, 79-90 (2011).

70. Schmid, M. \& Jensen, T. H. The exosome: a multipurpose RNA-decay machine. Trends Biochem. Sci. 33, 501-510 (2008).

71. Wlotzka, W., Kudla, G., Granneman, S. \& Tollervey, D. The nuclear RNA polymerase II surveillance system targets polymerase III transcripts. EMBO J. 30, 1790-1803 (2011).

72. Thompson, D. M. \& Parker, R. Stressing out over tRNA cleavage. Cell 138, 215-219 (2009).

73. Thompson, D. M., Lu, C., Green, P. J. \& Parker, R. tRNA cleavage is a conserved response to oxidative stress in eukaryotes. RNA 14, 2095-2103 (2008).

74. Thompson, D. M. \& Parker, R. The RNase Rny1p cleaves tRNAs and promotes cell death during oxidative stress in Saccharomyces cerevisiae. J. Cell Biol. 185, 43-50 (2009).

75. Luhtala, N. \& Parker, R. Structure-function analysis of Rny1 in tRNA cleavage and growth inhibition. PLOS ONE 7, e41111 (2012).

76. Hanada, T. et al. CLP1 links tRNA metabolism to progressive motor-neuron loss. Nature 495, 474-480 (2013).

77. Yamasaki, S., Ivanov, P., Hu, G.-F. \& Anderson, P. Angiogenin cleaves tRNA and promotes stressinduced translational repression. J. Cell Biol. 185, 35-42 (2009).

78. Dewe, J. M., Whipple, J. M., Chernyakov, I., Jaramillo, L. N. \& Phizicky, E. M. The yeast rapid tRNA decay pathway competes with elongation factor $1 \mathrm{~A}$ for substrate tRNAs and acts on tRNAs lacking one or more of several modifications. RNA 18, 1886-1896 (2012).

79. Chernyakov, I., Whipple, J. M., Kotelawala, L., Grayhack, E. J. \& Phizicky, E. M. Degradation of several hypomodified mature tRNA species in Saccharomyces cerevisiae is mediated by Met22 and the 5'-3' exonucleases Rat1 and Xrn1. Genes Dev. 22, 1369-1380 (2008).

80. Johnson, A. W. Rat1p and Xrn1p are functionally interchangeable exoribonucleases that are restricted to and required in the nucleus and cytoplasm, respectively. Mol. Cell. Biol. 17, 61226130 (1997). 
81. Sasikumar, A. N., Perez, W. B. \& Kinzy, T. G. The many roles of the eukaryotic elongation factor 1 complex. Wiley Interdiscip Rev RNA 3, 543-555 (2012).

82. Wilusz, J. E., Whipple, J. M., Phizicky, E. M. \& Sharp, P. A. tRNAs marked with CCACCA are targeted for degradation. Science 334, 817-821 (2011).

83. Oficjalska-Pham, D. et al. General repression of RNA polymerase III transcription is triggered by protein phosphatase type 2A-mediated dephosphorylation of Maf1. Mol. Cell 22, 623-632 (2006).

84. Huber, A. et al. Characterization of the rapamycin-sensitive phosphoproteome reveals that Sch9 is a central coordinator of protein synthesis. Genes Dev. 23, 1929-1943 (2009).

85. Moir, R. D. et al. Protein kinase A regulates RNA polymerase III transcription through the nuclear localization of Maf1. Proc. Natl. Acad. Sci. U.S.A. 103, 15044-15049 (2006).

86. Pluta, K. et al. Maf1p, a negative effector of RNA polymerase III in Saccharomyces cerevisiae. Mol. Cell. Biol. 21, 5031-5040 (2001).

87. Turowski, T. W. The impact of transcription on posttranscriptional processes in yeast. Gene (2013). doi:10.1016/j.gene.2013.04.021

88. Huynh, L.-N. et al. Linking tRNA localization with activation of nutritional stress responses. Cell Cycle 9, 3112-3118 (2010).

89. Quan, X., Yu, J., Bussey, H. \& Stochaj, U. The localization of nuclear exporters of the importinbeta family is regulated by Snf1 kinase, nutrient supply and stress. Biochim. Biophys. Acta 1773, 1052-1061 (2007).

90. Chan, C. T. Y. et al. A quantitative systems approach reveals dynamic control of tRNA modifications during cellular stress. PLoS Genet. 6, e1001247 (2010).

91. Pleiss, J. A., Whitworth, G. B., Bergkessel, M. \& Guthrie, C. Rapid, transcript-specific changes in splicing in response to environmental stress. Mol. Cell 27, 928-937 (2007).

92. Qiu, H. et al. Defects in tRNA processing and nuclear export induce GCN4 translation independently of phosphorylation of the alpha subunit of eukaryotic translation initiation factor 2. Mol. Cell. Biol. 20, 2505-2516 (2000).

93. Ghavidel, A. et al. Impaired tRNA nuclear export links DNA damage and cell-cycle checkpoint. Cell 131, 915-926 (2007). 


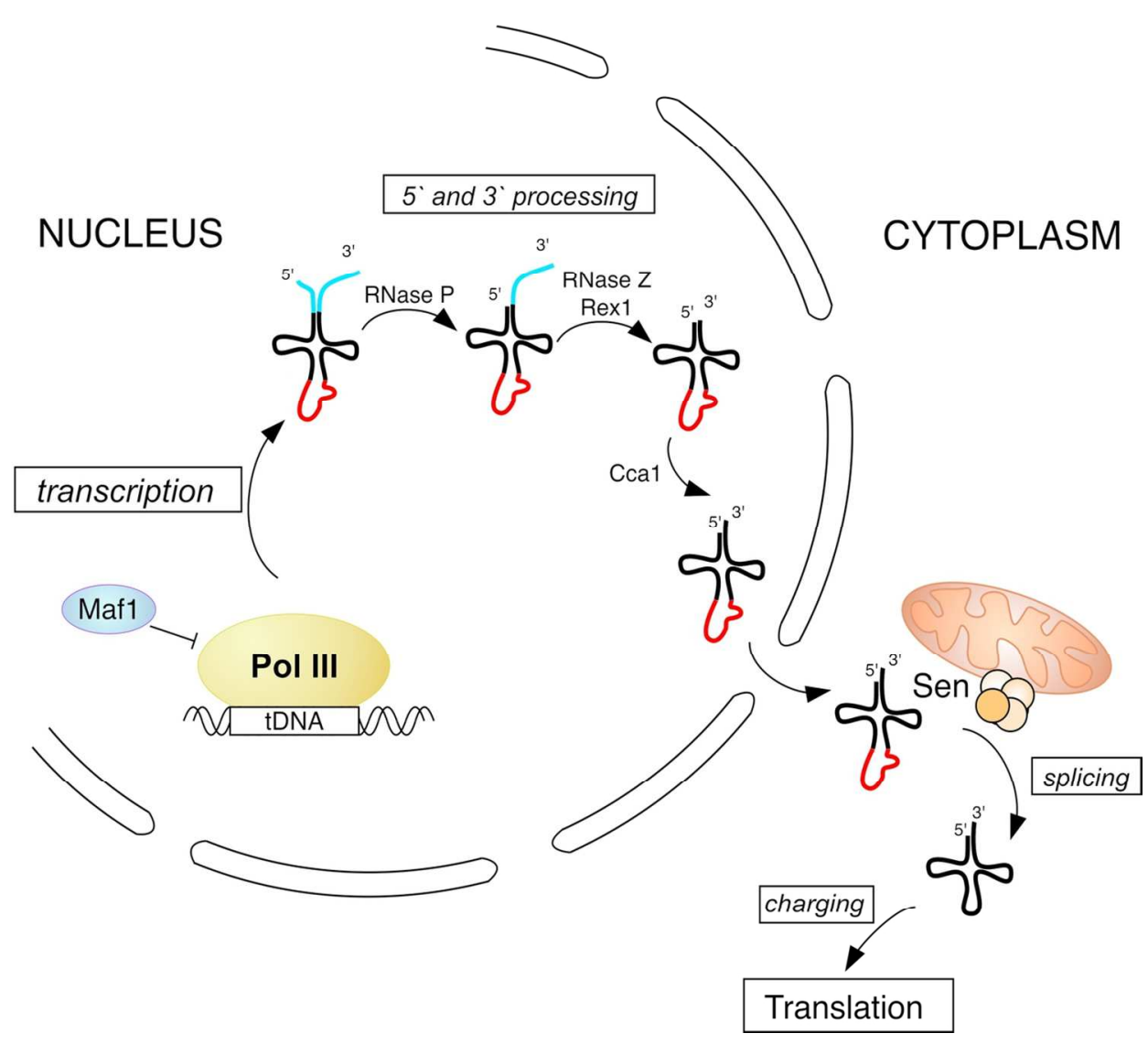

Figure 1. Maturation of intron containing tRNA. After transcription by Pol III subsequent steps of end maturation take place in the nucleus, next precursor is exported to the cytoplasm where intron is spliced, tRNA can be charged and directed to further processes. Enzymes responsible for each processing step are listed above corresponding arrows. Modifications which can be added in each step of tRNA biosynthesis are not presented on the scheme. $124 \times 118 \mathrm{~mm}(300 \times 300 \mathrm{DPI})$ 


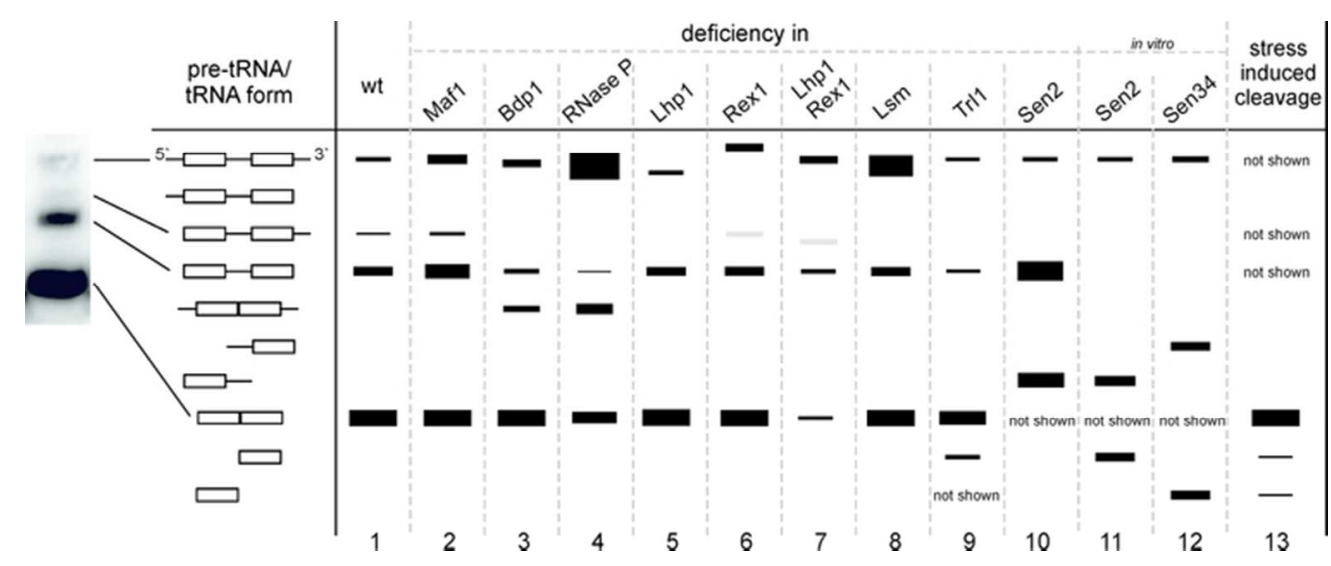

Figure 2. Representation of forms and fragments of precursors and mature tRNAs that accumulated or appeared in yeast mutants or under specific conditions. An example of Northern analysis of tRNAPhe(GAA)

in a wild type strain is given on the left. Bands on the blot represent unprocessed initial transcripts (designated ), 5 ' end matured intron-containing pre-tRNAs (designated ), end-matured intron-containing pre-tRNAs (designated ) and mature tRNAs (designated ). A graphical version of Northern blots reported previously for mutants defective in tRNA biosynthesis displays various intermediate forms designated by the respective symbols. Statement "not shown" is used to underline the forms that are not visualised by the given probes on Northern blots from cited articles. The band size is proportional to the wild type, in exception of those that are from in vitro studies. Each lane represents pre-tRNA and tRNA forms and the amount of each found in defined mutants as following: Maf1 -tRNAPhe(GAA) in maf1 $\triangle 50$; Bdp1-

tRNAIle(UAU) in bdp1 $\triangle 253-26949$; RNase P - tRNALeu(CAA) at $38^{\circ} \mathrm{C}$ in RPR1 cDNA dimer25; Lhp1tRNATyr (GUA) in Ihp1 $\triangle 44$; Rex1- tRNALys(UUU) in rex1 $\triangle 44$; Lhp1 and Rex1- tRNALys(UUU) in Ihp1 rex1 $\triangle 44$; Lsm- tRNALeu(CAA) after $6 \mathrm{~h}$ in glucose in GAL: : Ism335; Sen2- tRNALeu(CAA) in sen2-352; Sen2tRNATyr in Sen2 (His297Ala), in vitro study53; Sen34- tRNATyr in Sen34 (His217Ala) in vitro study53, Trl1tRNALeu(CAA) in trl1-4 with empty vector at $37^{\circ} \mathrm{C} 51$, stress induced cleavage- tRNAHis(GTG) in hts1.1 after $15 \mathrm{~min}$ at $39^{\circ} \mathrm{C} 73$. $64 \times 25 \mathrm{~mm}(300 \times 300$ DPI $)$ 


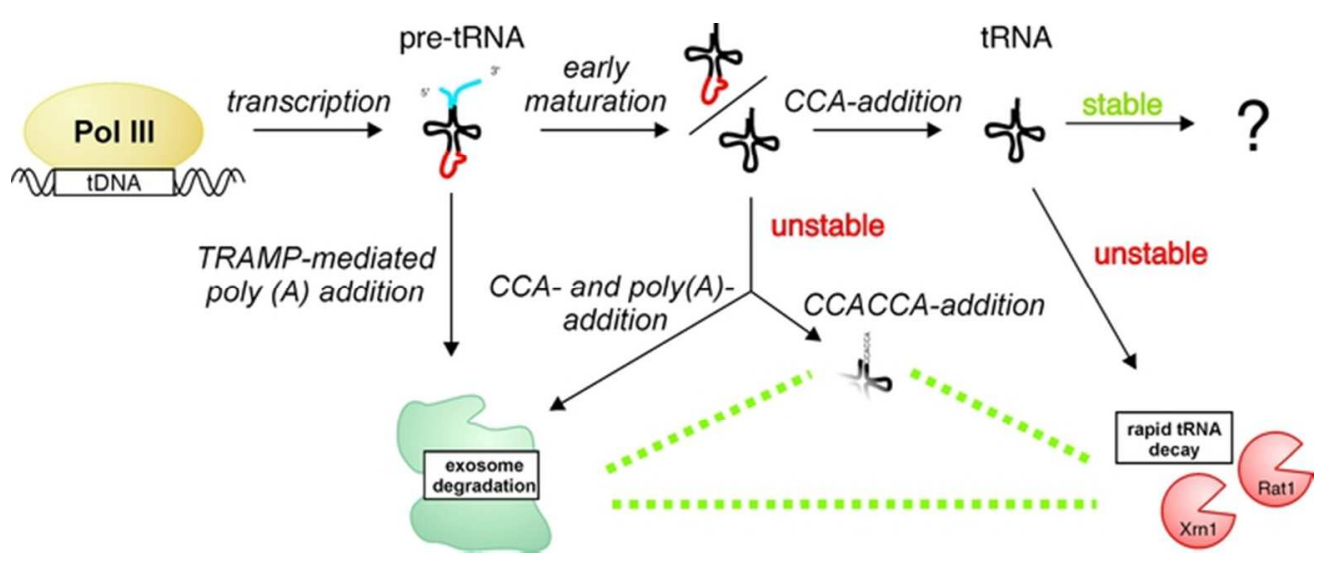

Figure 3. Current model of degradation during tRNA lifecycle. Newly transcribed tRNA precursor (pre-tRNA) can be polyadenylated on the $3^{\prime}$ end by TRAMP (Trf4/Air2/Mtr4 polyadenylation) complex and targeted to degradation by exosome. During early steps of processing defective intermediate can undergo exosomal degradation after marking with poly A tail. If the particle is unstable it can be marked with additional CCA sequence and directed to the Rapid tRNA Decay (RTD) pathway and/or polyadenylated and degraded by the exosome. As relationships of additional CCA marking of tRNA with RTD or exosome and correlation between exosome and RTD are unclear, they are marked on the scheme with dashed green lines. Lack of some modifications or mutations causing structural defects of mature tRNA results in instability of tRNA particle which is directed to RTD pathway. How mature stable tRNA is directed to degradation is still unknown. $61 \times 25 \mathrm{~mm}(300 \times 300$ DPI $)$ 


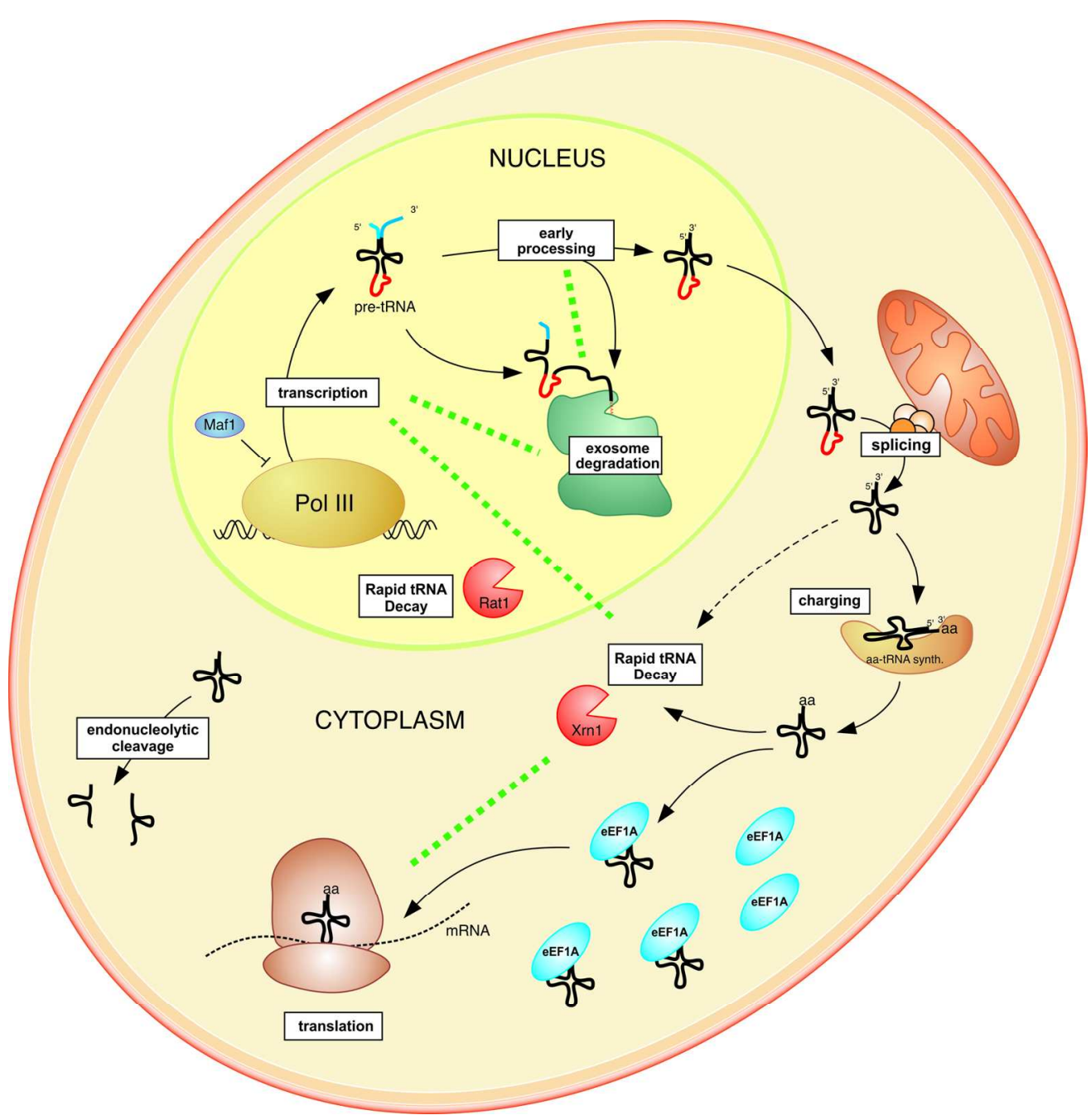

Figure 4. An interplay between tRNA biosynthesis and degradation in yeast. Primary tRNA transcript is synthesised by RNA Pol III which is regulated by Maf1 protein. Following the initial processing steps in the nucleus, where the $5^{\prime}$ leader and $3^{\prime}$ trailer are removed, the tRNA precursor is moved to the cytoplasm. CCA on the $3^{\prime}$ terminus and some modifications are added to the tRNA precursor. Introns are spliced on the outer surface of the mitochondrial membrane. tRNA is charged by the tRNA synthetase, bound by elongation factor (eEF1A) and delivered to the ribosome for translation. Mature tRNA under stress can be cleaved into tRNA halves. Turnover of tRNA is controlled by several pathways. In the nucleus, pre-tRNA can be degraded by the exosome complex or subjected to rapid tRNA decay (RTD) with Rat1 exonuclease. In the cytoplasm, mature tRNAs can be directed to cytoplasmic RTD by Xrn1 exonuclease. Uncovered/emerging interactions between transcription, processing and decay pathways, which are described in detail in the text, are marked with green dashed lines. $157 \times 160 \mathrm{~mm}(300 \times 300$ DPI $)$ 